\title{
SOME REMARKS ON MILOVANOVIĆ-PEČARIĆ INEQUALITY AND APPLICAITONS FOR SPECIAL MEANS AND NUMERLCAL INTEGRATIONS
}

\author{
S. S. DRAGOMIR, Y. J. CHO AND S. S. KIM
}

\begin{abstract}
In this paper, we shall give some applications of the Milovanović-Peçarić inequality for special means in numerical integrations and prove some new inequalities of the MilovanovićPečarić type in which the bound will be expressed in terms of the first derivatives.
\end{abstract}

\section{Introduction}

In 1976, G. V. Milovanović and J. E. Pečarić proved the following inequality of Ostrowski's type (see, for example, (1, p. 468]):

$$
\begin{aligned}
& \left|\frac{1}{2}[(b-a) f(x)+(x-a) f(a)+(b-x) f(b)]-\int_{a}^{b} f(t) d t\right| \\
\leq & \frac{\left\|f^{\prime \prime}\right\|_{\infty}(b-a)^{3}}{4}\left[\frac{1}{12}+\frac{\left(x-\frac{a+b}{2}\right)^{2}}{(b-a)^{2}}\right]
\end{aligned}
$$

for all $x \in[a, b]$, provided that $f:[a, b] \rightarrow R$ is twice differentiable on (a,b) and $\left\|f^{\prime \prime}\right\|_{\infty}=$ $\sup _{t \in(a, b)}\left|f^{\prime \prime}(t)\right|<\infty$.

If, in the above inequality, we choose $x=\frac{a+b}{2}$, then we have

$$
\left|\frac{1}{2}\left[(b-a) f\left(\frac{a+b}{2}\right)+(b-a) \frac{f(a)+f(b)}{2}\right]-\int_{a}^{b} f(t) d t\right| \leq \frac{\left\|f^{\prime \prime}\right\|_{\infty}(b-a)^{3}}{48}
$$

and, if we choose $x=a$ or $x=b$, then we have the following trapezoid inequality:

$$
\left|(b-a) \frac{f(a)+f(b)}{2}-\int_{a}^{b} f(t) d t\right| \leq \frac{\left\|f^{\prime}\right\|_{\infty}(b-a)^{3}}{12} .
$$

Received November 5, 1998.

1991 Mathematics Subject Classification. 26B15, 26B20

Key words and phrases. Milovanović-Pečarić inequality, Ostrowski's inequality. 
In what follows we shall point out some applications of the inequality (1.1) for special means in numerical integraitons as well as we shall prove some new inequalities of the Milovanović-Pečarić type in which the bound will be expressed in terms of the first derivatives of $f$.

\section{Applications for Special Means}

Let us reall the following concepts we shall use in sequel:

(a) The arithmetric mean: $A=A(a, b)=(a+b) / 2, \quad a, b \geq 0$,

(b) The geometric mean: $G=G(a, b)=\sqrt{a b}, \quad a, b \geq 0$,

(c) The harmonic mean: $H=H(a, b)=2 /\left(\frac{1}{a}+\frac{1}{b}\right), \quad a, b>0$,

(d) The logarithmic mean:

$$
L=L(a, b)= \begin{cases}a & \text { if } a=b \\ \frac{b-a}{\ln b-\ln a} & \text { if } a \neq b, a, b>0 .\end{cases}
$$

(e) The identric mean:

$$
I=I(a, b)= \begin{cases}a & \text { if } a=b \\ \frac{1}{e}\left(\frac{b^{b}}{a^{a}}\right)^{\frac{1}{b-a}} & \text { if } a \neq b, a, b>0 .\end{cases}
$$

It is well-known in the literature that

$$
H \leq G \leq L \leq I \leq A
$$

In what follows, we shall use the inequality (1.1) in the following version:

$$
\begin{aligned}
& \left|\frac{1}{b-a} \int_{a}^{b} f(y) d y-\frac{1}{2}\left[f(x)+\frac{b f(b)-a f(a)}{b-a}-x \cdot \frac{f(b)-f(a)}{b-a}\right]\right| \\
\leq & \frac{\left\|f^{\prime \prime}\right\|_{\infty}(b-a)^{2}}{4}\left[\frac{1}{12}+\frac{\left(x-\frac{a+b}{2}\right)^{2}}{(b-a)^{2}}\right]
\end{aligned}
$$

for all $x \in[a, b]$.

Example 1. Consider the mapping $f(x)=\frac{1}{x}$ for all $x \in[a, b] \subset(0, \infty)$. Then we have

$$
\begin{gathered}
\frac{1}{b-a} \int_{a}^{b} f(y) d y=L^{-1}, \quad \frac{b f(b)-a f(a)}{b-a}=0, \quad \frac{f(b)-f(a)}{b-a}=-\frac{1}{G^{2}}, \\
f^{\prime}(x)=-\frac{1}{x^{2}}, \quad f^{\prime \prime}(x)=\frac{2}{x^{3}}, \quad\left\|f^{\prime \prime}\right\|_{\infty}=\frac{2}{a^{3}} .
\end{gathered}
$$

Therefore, the inequality (2.2) becomes

$$
\left|L^{-1}-\frac{1}{2}\left[x^{-1}+\frac{x}{G^{2}}\right]\right| \leq \frac{2(b-a)^{2}}{4 a^{3}}\left[\frac{1}{12}+\frac{(x-A)^{2}}{(b-a)^{2}}\right],
$$


which is equivalent to the following:

$$
0 \leq \frac{1}{L}-\frac{G^{2}+x^{2}}{2 x G^{2}} \leq \frac{(b-a)^{2}}{2 a^{3}}\left[\frac{1}{12}+\frac{(x-A)^{2}}{(b-a)^{2}}\right] .
$$

Now, choosing $x=G$ in (2.4), we have

$$
0 \leq L-G \leq \frac{(b-a)^{2}}{2 a^{3}}\left[\frac{1}{12}+\frac{(A-G)^{2}}{(b-a)^{2}}\right] L G
$$

If we choose $x=L$ in (2.4), we have

$$
\begin{aligned}
0 & \leq L^{2}-G^{2} \\
& \leq \frac{(b-a)^{2}}{a^{3}}\left[\frac{1}{12}+\frac{(L-A)^{2}}{(b-a)^{2}}\right] L G^{2} \\
& =\frac{b}{a^{2}}(b-a)^{2}\left[\frac{1}{12}+\frac{(L-A)^{2}}{(b-a)^{2}}\right] L .
\end{aligned}
$$

If we choose $x=A$ in (2.4), we have

$$
0 \leq G^{2}+A^{2}-2 A G \frac{G}{L} \leq \frac{A G^{2}(b-a)^{2}}{12 a^{3}} .
$$

Example 2. Consider the mapping $f:[a, b] \rightarrow R$ defined by $f(x)=\ln x$ for all $x \in[a, b] \subset(0, \infty)$. Then we have

$$
\begin{gathered}
\frac{1}{b-a} \int_{a}^{b} f(y) d y=\ln I, \quad \frac{b f(b)-a f(a)}{b-a}=\ln (e I), \quad \frac{f(b)-f(a)}{b-a}=L^{-1}, \\
f^{\prime}(x)=\frac{1}{x}, \quad f^{\prime \prime}(x)=-\frac{1}{x^{2}}, \quad\left\|f^{\prime \prime}\right\|_{\infty}=\frac{1}{a^{2}}
\end{gathered}
$$

and then the equality (2.2) becomes

$$
\left|\ln I-\frac{1}{2}\left[\ln x+\ln (e I)-\frac{x}{L}\right]\right| \leq \frac{(b-a)^{2}}{4 a^{2}}\left[\frac{1}{12}+\frac{(A-x)^{2}}{(b-a)^{2}}\right]
$$

that is,

$$
\left|\ln \left(\frac{x}{I}\right)-\frac{x}{L}+1\right| \leq \frac{(b-a)^{2}}{2 a^{2}}\left[\frac{1}{12}+\frac{(A-x)^{2}}{(b-a)^{2}}\right] .
$$

We know that, for all $x>0, \frac{x}{L} \geq \frac{x}{1}$ and, for all $y>0, y-1 \geq \ln y$ and so $\frac{x}{L}-1 \geq \ln \frac{x}{I} \geq \ln \frac{x}{1}$ and the above inequality becomes

$$
0 \leq \frac{x}{L}-1-\ln \left(\frac{x}{I}\right) \leq \frac{(b-a)^{2}}{2 a^{2}}\left[\frac{1}{12}+\frac{(A-x)^{2}}{(b-a)^{2}}\right]
$$

for all $x \in[a, b] \subset(0, \infty)$. 
If we choose $x=L$ in (2.8), we have

$$
1 \leq \frac{I}{L} \leq \exp \left[\frac{(b-a)^{2}}{2 a^{2}}\left[\frac{1}{12}+\frac{(A-L)^{2}}{(b-a)^{2}}\right]\right]
$$

If we choose $x=I$ in $(2.8)$, we have

$$
0 \leq I-L \leq \frac{(b-a)^{2}}{2 a^{2}}\left[\frac{1}{12}+\frac{(A-I)^{2}}{(b-a)^{2}}\right] L
$$

Finally, if we choose $x=A$ in (2.8), then we have

$$
0 \leq \frac{A}{L}-1-\ln \left(\frac{A}{I}\right) \leq \frac{(b-a)^{2}}{24 a^{2}}
$$

\section{Applications in Numerical Integrations}

We shall apply the inequality (1.1) to obtain some quadrature formulas which are similar with the trapezoid and mid-point quadrature rules (see [1]).

Theorem 3.1. Let $f:[a, b] \rightarrow R$ be a twice differentiable mapping on $(a, b)$ with $\left\|f^{\prime \prime}\right\|_{\infty}=\sup _{t \in(a, b)}\left|f^{\prime \prime}(t)\right|<\infty$. Then, for any partition $I_{h}: a=x_{0}<x_{1}<\cdots<$ $x_{n-1}<x_{n}=b$ of $[a, b]$ and any intermediate point vectors $\xi=\left(\xi_{0}, \xi_{1}, \cdots, \xi_{n-1}\right)$ satisfying $\xi_{i} \in\left[x_{i}, x_{i+1}\right](i=0,1, \ldots, n-1)$, we have

$$
\int_{a}^{b} f(x) d x=A_{M, P}\left(f, I_{h}, \xi\right)+R_{M, P}\left(f, I_{h}, \xi\right)
$$

where $A_{M, P}\left(f, I_{h}, \xi\right)$ is a generalization of the Riemann sum as follow:

$$
A_{M, P}\left(f, I_{h}, \xi\right)=\frac{1}{2}\left[\sum_{i=0}^{n-1} f\left(\xi_{i}\right) h_{i}+\sum_{i=0}^{n-1}\left(\xi_{i}-x_{i}\right) f\left(x_{i}\right)+\sum_{i=0}^{n-1}\left(x_{i+1}-\xi_{i}\right) f\left(x_{i+1}\right)\right]
$$

where the remainder term $R_{M, P}\left(f, I_{h}, \xi\right)$ satisfies the estimation

$$
\begin{aligned}
\left|R_{M, R}\left(f, I_{h}, \xi\right)\right| & \leq \frac{\left\|f^{\prime \prime}\right\|_{\infty}}{4} \sum_{i=0}^{n-1} h_{i}^{2}\left[\frac{1}{12}+\frac{\left(\xi_{i}-\frac{x_{i}+x_{i}+1}{2}\right)^{2}}{h_{i}^{2}}\right] \\
& \leq \frac{\left\|f^{\prime \prime}\right\|_{\infty}}{12} \sum_{i=0}^{n-1} h_{i}^{2}
\end{aligned}
$$

where $h_{i}=x_{i+1}-x_{i}$. 
Proof. Applying the inequality (1.1) on the interval $\left[x_{i}, x_{i+1}\right](i=0,1, \ldots, n-1)$, we have

$$
\begin{aligned}
& \left|\int_{x_{i}}^{x_{i+1}} f(t) d t-\frac{1}{2}\left[f\left(\xi_{i}\right) h_{i}+\left(\xi_{i}-x_{i}\right) f\left(x_{i}\right)+\left(x_{i+1}-\xi_{i}\right) f\left(x_{i+1}\right)\right]\right| \\
\leq & \frac{\left\|f^{\prime \prime}\right\|_{\infty} h_{i}^{2}}{4}\left[\frac{1}{12}+\frac{\left(\xi_{i}-\frac{x_{i}+x_{i+1}}{2}\right)^{2}}{h_{i}^{2}}\right] \\
\leq & \frac{\left\|f^{\prime \prime}\right\|_{\infty} h_{i}^{2}}{12}
\end{aligned}
$$

as $\left(\xi_{i}-\frac{x_{i}+x_{i+1}}{2}\right)^{2} \leq \frac{h_{i}^{2}}{4}$. Summing over $i$ from 0 to $n-1$, we deduce easily the estimation (3.3).

Corollary 3.2. With the above assumptions, we have

$$
\int_{a}^{b} f(x) d x=A_{M, P}\left(f, I_{h}, \xi^{*}\right)+R_{M, P}\left(f, I_{h}, \xi^{*}\right)
$$

where

$$
A_{M, P}\left(f, I_{h}, \xi^{*}\right)=\frac{1}{2}\left[\sum_{i=0}^{n-1} f\left(\frac{x_{i}+x_{i+1}}{2}\right) h_{i}+\sum_{i=0}^{n-1} \frac{f\left(x_{i}\right)+f\left(x_{i+1}\right)}{2} h_{i}\right]
$$

is a mixture between the trapezoid and mid-point quadrature rules and the remainder satisfies the following inequality:

$$
\left|R_{M, P}\left(f, I_{h}, \xi^{*}\right)\right| \leq \frac{\left\|f^{\prime \prime}\right\|_{\infty}}{48} \sum_{i=0}^{n-1} h_{i}^{3}
$$

\section{Applications of Iyengar's Inequality}

In 1938, K. S. K. Iyengar [1], by means of geometrical condition, has proved the following theorem:

Theorem 4.1. Let $f$ be a nonconstant differentiable function on $[a, b]$ and assume that $\left\|f^{\prime}\right\|_{\infty}=\sup _{t \in(a, b)}\left|f^{\prime}(t)\right|<\infty$. Then

$$
\left|\int_{a}^{b} f(x) d x-\frac{1}{2}(b-a)(f(a)+f(b))\right| \leq \frac{\left\|f^{\prime}\right\|_{\infty}(b-a)^{2}}{4}-\frac{1}{4\left\|f^{\prime}\right\|_{\infty}}(f(b)-f(a))^{2} .
$$

In this section, by the use of Iyengar's theorem, we shall point out an inequality of the Milovanović-Pečarić type. 
Theorem 4.2. With the above assumptions on the mapping $f$, we have the following inequality:

$$
\begin{aligned}
& \left|\int_{a}^{b} f(t) d t-\frac{1}{2}[f(x)(b-a)+(x-a) f(a)+(b-x) f(b)]\right| \\
\leq & \frac{M}{4}\left[\frac{1}{4}+\frac{\left(x-\frac{a+b}{2}\right)^{2}}{(b-a)^{2}}\right](b-a)^{2}-\frac{1}{8 M}(f(b)-f(a))^{2}
\end{aligned}
$$

for all $x \in[a, b]$, where $M=\left\|f^{\prime}\right\|_{\infty}<\infty$.

Proof. Applying (4.1) on the intervals $[a, x]$ and $[x, b]$, we have

$$
\left|\int_{a}^{x} f(t) d t-\frac{1}{2}(x-a)(f(a)+f(x))\right| \leq \frac{M(x-a)^{2}}{4}-\frac{1}{4 M}(f(x)-f(a))^{2}
$$

and

$$
\left|\int_{x}^{b} f(t) d t-\frac{1}{2}(b-x)(f(b)+f(x))\right| \leq \frac{M(b-x)^{2}}{4}-\frac{1}{4 M}(f(b)-f(x))^{2} .
$$

Summing the above two inequalities, we have

$$
\begin{aligned}
& \left|\int_{a}^{b} f(t) d t-\frac{1}{2}[(b-a) f(x)+(x-a) f(a)+(b-x) f(b)]\right| \\
\leq & \frac{M}{4}\left[(b-x)^{2}+(x-a)^{2}\right]-\frac{1}{4 M}\left[(f(x)-f(a))^{2}+(f(b)-f(x))^{2}\right] .
\end{aligned}
$$

Now, by a simple calculation, we have

$$
(b-x)^{2}+(x-a)^{2}=(b-a)^{2}\left[\frac{1}{4}+\frac{\left(x-\frac{a+b}{2}\right)^{2}}{(b-a)^{2}}\right]
$$

and

$$
2\left[(f(x)-f(a))^{2}+(f(b)-f(x))^{2}\right] \geq(f(b)-f(a))^{2} .
$$

Therefore, (4.3) gives us the desired inequality (4.2).

Corollary 4.3. With the above assumptions, we have

$$
\begin{aligned}
& \left|\int_{a}^{b} f(t) d t-\frac{1}{2}\left[f\left(\frac{a+b}{2}\right)+\frac{f(a)+f(b)}{2}\right](b-a)\right| \\
\leq & \frac{M}{16}(b-a)^{2}-\frac{1}{8 M}(f(b)-f(a))^{2} .
\end{aligned}
$$


In what follows, we shall consider the following weaker version of (4.2) which has nice applications for special means and in Numerical Analysis:

$$
\begin{aligned}
& \left|\frac{1}{b-a} \int_{a}^{b} f(y) d y-\frac{1}{2}\left[f(x)+\frac{b f(b)-a f(a)}{(b-a)}-x \cdot \frac{f(b)-f(a)}{b-a}\right]\right| \\
\leq & \frac{\left\|f^{\prime}\right\|_{\infty}}{4}\left[\frac{1}{4}+\frac{\left(x-\frac{a+b}{2}\right)^{2}}{(b-a)^{2}}\right](b-a)
\end{aligned}
$$

for all $x \in[a, b]$.

Example 3. Consider the mapping $f(x)=\frac{1}{x}$ for all $x \in[a, b] \subset(0, \infty)$. Then, as in Section 2, we have the following inequalities:

$$
0 \leq \frac{1}{L}-\frac{G^{2}+x^{2}}{2 x G^{2}} \leq \frac{(b-a)^{2}}{4 a^{2}}\left[\frac{1}{4}+\frac{(x-A)^{2}}{(b-a)^{2}}\right]
$$

for all $x \in[a, b]$. Now, choosing $x=G$ in (4.6), we have

$$
0 \leq L-G \leq \frac{(b-a)}{4 a^{2}}\left[\frac{1}{4}+\frac{(x-A)^{2}}{(b-a)^{2}}\right] L G .
$$

If we choose $x=A$ in (4.6), we have

$$
0 \leq G^{2}+A^{2}-2 A G \frac{G}{L} \leq \frac{(b-a) A L G^{2}}{8 a^{2}} .
$$

Now, let observe that in (2.7) we got the bound $\frac{A L G^{2}(b-a)^{2}}{12 a^{3}}$ for $a^{2}+A^{2}-2 A G \frac{G}{L}$. Consider the ratio

$$
R=\left[\frac{A L G^{2}(b-a)^{2}}{12 a^{3}}\right] /\left[\frac{(b-a) A L G^{2}}{8 a^{2}}\right]=\frac{2(b-a)}{3 a} .
$$

Now, if $2 b \geq 5 a$, then the estimate in (4.8) is better than the estimation provided by (2.2).

Example 4. Let consider the mapping $f:[a, b] \rightarrow R$ defined by $f(x)=\ln x$ for all $x \in[a, b] \subset(0, \infty)$. Then we have (see Section 2)

$$
0 \leq \frac{x}{L}-1-\ln \left(\frac{x}{I}\right) \leq \frac{(b-a)}{4 a}\left[\frac{1}{4}+\frac{(x-A)^{2}}{(b-a)^{2}}\right] .
$$

If we choose $x=L$ in (4.9), we have

$$
1 \leq \frac{I}{L} \leq \exp \left[\frac{(b-a)}{4 a}\left[\frac{1}{4}+\frac{(A-L)^{2}}{(b-a)^{2}}\right]\right] .
$$


If we choose $x=I$ in (4.9), we have

$$
0 \leq I-L \leq \frac{(b-a)}{4 a}\left[\frac{1}{4}+\frac{(A-I)^{2}}{(b-a)^{2}}\right] .
$$

Finally, we shall apply (4.5) to obtain some quadrature formulas which are similar with the trapeziod and mid-point quadreture rules.

Theorem 4.4. Let $f:[a, b] \rightarrow R$ be a differentiable mapping on $(a, b)$ with $\left\|f^{\prime}\right\|_{\infty}=$ $\sup _{i \in(a, b)}\left|f^{\prime}(t)\right|<\infty$. Then, for any partition $I_{h}: a=x_{0}<x_{1}<\cdots<x_{n-1}<$ $x_{n}=b$ of $[a, b]$ and any intermediate point vectors $\xi=\left(\xi_{0}, \xi_{1}, \ldots, \xi_{n-1}\right)$ satisfying $\xi_{i} \in$ $\left[x_{i}, x_{i+1}\right](i=0,1, \ldots, n-1)$, we have

$$
\int_{a}^{b} f(x) d x=A_{M . P}\left(f, I_{h}, \xi\right)+R_{M, P}\left(f, I_{h}, \xi\right),
$$

where $A_{M, P}\left(f, I_{h}, \xi\right)$ is a generalization of the Riemann sum as follow:

$$
A_{M, P}\left(f, I_{h}, \xi\right)=\frac{1}{2}\left[\sum_{i=0}^{n-1} f\left(\xi_{i}\right) h_{i}+\sum_{i=0}^{n-1}\left(\xi_{i}-x_{i}\right) f\left(x_{i}\right)+\sum_{i=0}^{n-1}\left(x_{i+1}-\xi_{i}\right) f\left(x_{i+1}\right)\right]
$$

and the remainder term $R_{M, P}\left(f, I_{h}, \xi\right)$ satisfies the following estimation:

$$
\begin{aligned}
\left|R_{M, R}\left(f, I_{h}, \xi\right)\right| & \leq \frac{\left\|f^{\prime}\right\|_{\infty}}{4} \sum_{i=0}^{n-1}\left[\frac{1}{4}+\frac{\left(\xi_{i}-\frac{x_{i}+x_{i+1}}{2}\right)^{2}}{h_{i}^{2}}\right] h_{i}^{2} \\
& \leq \frac{\left\|f^{\prime}\right\|_{\infty}}{4} \sum_{i=0}^{n-1} h_{i}^{2} .
\end{aligned}
$$

Proof. Applying the inequality (4.5) on the interval $\left[x_{i}, x_{i+1}\right](i=0,1, \ldots, n-1)$, we have

$$
\begin{aligned}
& \left|\int_{x_{i}}^{x_{i+1}} f(t) d t-\frac{1}{2}\left[f\left(\xi_{i}\right) h_{i}+\left(\xi_{i}-x_{i}\right) f\left(x_{i}\right)+\left(x_{i+1}-\xi_{i}\right) f\left(x_{i+1}\right)\right]\right| \\
\leq & \frac{\left\|f^{\prime}\right\|_{\infty}}{4}\left[\frac{1}{4}+\frac{\left(\xi_{i}-{\frac{x_{i}+x_{i+1}}{2}}^{2}\right.}{h_{i}^{2}}\right] h_{i}^{2} \leq \frac{\left\|f^{\prime}\right\|_{\infty}}{4}\left[\frac{1}{4}+\frac{1}{4}\right] \frac{h_{i}^{2}}{4} \\
= & \frac{\left\|f^{\prime}\right\|_{\infty}}{8} h_{i}^{2} .
\end{aligned}
$$

Summing over $i$ from 0 to $n-1$, we deduce easily the estimation (4.14).

Corollary 4.5. With the above assumptions, we have

$$
\int_{a}^{b} f(x) d x=A_{M, P}\left(f, I_{h}, \xi^{*}\right)+R_{M, P}\left(f, I_{h}, \xi^{*}\right)
$$


where

$$
A_{M, P}\left(f, I_{h}, \xi^{*}\right)=\frac{1}{2}\left[\sum_{i=0}^{n-1} f\left(\frac{x_{i}+x_{i+1}}{2}\right) h_{i}+\sum_{i=0}^{n-1} \frac{f\left(x_{i}\right)+f\left(x_{i+1}\right)}{2} h_{i}\right]
$$

is a mixture between the trapezoid and mid-point quadrature rules and the remainder satisfies the following inequality:

$$
\left|R_{M, P}\left(f, I_{h}, \xi^{*}\right)\right| \leq \frac{\left\|f^{\prime}\right\|_{\infty}}{16} \sum_{i=0}^{n-1} h_{i}^{2}
$$

Remark 4.6. If the mapping $f$ is not twice differentiable on $(a, b)$ or the second derivative $f^{\prime \prime}$ is very large on $(a, b)$, we can not apply the quadrature formula (3.1) provided by the Milovanović-Pečarić inequality and thus (4.12) provides an alternative solution in terms of the first derivative.

\section{References}

[1] D. S. Mitrinović, J. E. Pečarić and A. M. Fink, Inequalities Involuing Functions and Their Integrals and Drivatives, Kluwer Academic Publishers, Dordrecht, 1991.

Department of Computer and Mathematical Science, Victoria University of Technology, P. O. Box 144.28, MCMC, Melbourne, Victoria 8001, Australia

Department of Mathematics, Gyeongsang National University, Chinju 660-701, Korea Department of Mathematics, Dongeui University, Pusan 614-714, Korea 